\title{
A Proposal of Predicting Formulae for Influence of Stress on Magnetostriction in Grain Oriented Silicon Steel
}

\author{
Hiroaki MASUI, Masato MIZOKAMI, Yukio MATSUO'1) and Hisashi MOGI'1) \\ Steel Research Laboratory, Nippon Steel Corporation, Shintomi, Futtsu, Chiba-ken, 293 Japan. \\ 1) Yawata Laboratory, Nippon Steel Corporation, Tobihata, Tobata-ku, Kitakyushu, Fukuoka-ken, 804 Japan.
}

(Received on October 20,1994; accepted in final form on December 16, 1994)

\begin{abstract}
Research has been carried out on the magnetostriction in grain oriented silicon steel, which is known to be one of main causes for noise problem and be crucially influenced by stress condition on the steel in either manufacturing or application process. Generalization of the formulation for the influence of the stress on the magnetostriction has been investigated, which enables computing design to evaluate an occurrence of the magnetostriction under various practical stress conditions, proposing a predictive formulae for the occurrence. The deteriorating influence on the magnetostriction of compressive stress applied to grain oriented silicon steel was attributed mainly to the formation of $90^{\circ}$ supplementary domains or triangular closure domains around grooves made on the surface. The supplementary domains or the triangular closure domains were found to occur under generalized condition that the strain elastic energy in easy magnetization axis [100], which is generally parallel to rolling direction in Goss orientation texture, is smaller than those in [010] and [001] (other easy magnetization axes). This result led to practical equation for the influence of the stress on the occurrence of the magnetostriction, having been substantiated by elaborate experimental results ranging from the effect of coating stress on the magnetostriction to the influence of residual internal tensile stress near scratched line on the surface. The obtained formulae were confirmed to be effective even for the magnetostriction under complicated state of superimpositon of bi-axial stresses experimented by Moses et al.
\end{abstract}

KEY WORDS: magnetostriction; strain elastic energy; grain oriented silicon steel; easy magnetization axis; superimposed stress; $90^{\circ}$ domain wall; coating stress; $90^{\circ}$ supplementary domain; compressive stress; trianglular closure domain.

\section{Introduction}

Grain oriented silicon steel which was invented by Goss $^{1)}$ and developed by many other researchers ${ }^{2-9)}$ as a low core loss material for electric transformers, has contributed to energy saving for industry and civilization as well. Recently, on the other hand, noise generated by transformers has become important from an environmental point of view and also in relation to the efficiency of energy consumption. In many causes of a noise emitted by transformer, magnetostriction in grain oriented silicon steel is recognized as a problem that inevitably confronts with transformer designer and is incessantly studied to be suppressed as lower as possible based upon long experience. As extensive data show, the magnetostriction in grain oriented silicon steel has been remarkably influenced by various stress conditions through manufacturing and application process of the steel not only until a construction of transformer but all time during its operation. The reason why the magnetostriction in grain oriented silicon steel is remarkably influenced by stress condition, such as the deterioration by compressive stress, has been elucidated by many researchers ${ }^{10-20}$ recently. Even with these studies, however, it was not always easy to estimate the influence of superimposed stresses on the magnetostriction. A - matter of first importance to industrial standpoint is to forecast an occurrence of the magnetostriction in grain oriented silicon steel more precisely and quantitatively even under any superimposed stress conditions attributed to process factors both in production of the steel and in fabrication, and operation of transformer as well. This study, first of all, aimes to derive a predictive equation of stress influence on the occurrence of the magnetostriction in grain oriented silicon steel by both hypothetical theory and elaborate experimental procedure, and introduce an application method of the equation with an exemplified procedure even upon practical superimposed stress condition. Based on the concept, it is referred to how a low magnetostriction grain oriented silicon steel shall be properly designed.

\section{Experimental Procedure}

Three $\%$ grain oriented silicon steel $(\mathrm{Hi}-\mathrm{B}, 0.23 \mathrm{~mm}$, B8:1.93 T) was prepared for experiments. Two kinds of surface coating stress conditions were made in such a way as one (sample A) with high tension insulation 
Table 1. Magnetic properties of the samples.

\begin{tabular}{|c|c|c|c|c|c|c|}
\hline \multirow{2}{*}{ Sample } & \multirow{2}{*}{$\begin{array}{c}\text { Thickness } \\
(\mathrm{mm})\end{array}$} & \multirow{2}{*}{$\begin{array}{c}\text { Coating } \\
\text { stress } \\
\text { (MPa) }\end{array}$} & \multicolumn{3}{|c|}{ Iron loss $(\mathrm{W} / \mathrm{kg})$} & \multirow{2}{*}{$\begin{array}{c}\text { Magnetic } \\
\text { induction } \\
(800 \mathrm{~A} / \mathrm{m}, \mathrm{T})\end{array}$} \\
\hline & & & $W_{13 / 50}$ & $W_{15 / 50}$ & $W_{17 / 50}$ & \\
\hline A & 0.23 & 14.7 & 0.43 & 0.58 & 0.80 & 1.930 \\
\hline B & 0.23 & 3.9 & 0.51 & 0.67 & 0.91 & 1.938 \\
\hline
\end{tabular}

coating film on mill glass and another (sample B) by dissolving off the high tension coating film from mill glass by alkali solution. Table 1 shows magnetic properties of the samples. Magnetostriction in longitudinal (rolling) direction was measured in a range of $0.5-1.9 \mathrm{~T}$ at $50 \mathrm{~Hz}$ by using a Doppler type magnetostriction tester simultaneously applying compressive stress from 0 to $3 \mathrm{MPa}$ along the rolling direction on $60 \times 300 \mathrm{~mm}$ specimens. Observation of magnetic domain without any compulsory magnetic field was done by a $200 \mathrm{KV}$ scanning electron microscope enclosing a special device, which applies compressive stress from zero to $8 \mathrm{MPa}$ to the rolling direction on $15 \times 70 \mathrm{~mm}$ specimen. Coating stress was measured by stripping off mill glass and, in sample $A$, the coating film as well, only on one side of specimen, combined with the aid of checking the coefficient of elasticity by precise tensile tester. Some specimens were scratched by using a ball-point pen with a pitch of $10 \mathrm{~mm}$ distance perpendicular to rolling direction with about $40 \mu \mathrm{m}$ contact width on the steel surface. In few specimen, $850^{\circ} \mathrm{C} \times 5 \mathrm{~h}$ stress relief annealing was conducted.

\section{Experimental Results}

\subsection{Magnetostriction}

Two kinds of the different surface coating stress samples were prepared. Sample A had a coating stress of $14.7 \mathrm{MPa}$, while sample $B$ showed $3.9 \mathrm{MPa}$. Magnetostriction (0-Peak, a difference from magnetostriction at maximum flux density $T_{\max }$ to that at 0 flux density $T_{0}$ ) at $1.7 \mathrm{~T}$ is shown in Fig. 1. With even small increase of compressive stress, elongation by magnetostriction in sample B became remarkably large, while extremely small increase of the elongation was perceived even on $3 \mathrm{MPa}$ compressive stress in sample A. Figure 2 gives a relation between magnetostriction and magnetic flux density. The magnetostriction increased enormously and proportionally with magnetic flux density in sample B. Similar tendency to both Figs. 1 and 2 was recognized in Peak-Peak (a difference between maximum magnetostriction and minimum one) measurements, too. Butterfly loops are shown in Fig. 3. As indicated in Fig. 1, increase of magnetostrictive elongation by scratch on surface is not negligible especially in sample $B$.

\subsection{Observation of Domain by Scanning Electron Mi- croscope}

Observation of magnetic domain by scanning electron microscope under the stress condition was exemplified in Fig. 4 (Sample A) and Fig. 5 (Sample B). In stress free condition of sample $\mathrm{A}$, exclusively main domains $\left(180^{\circ}\right.$

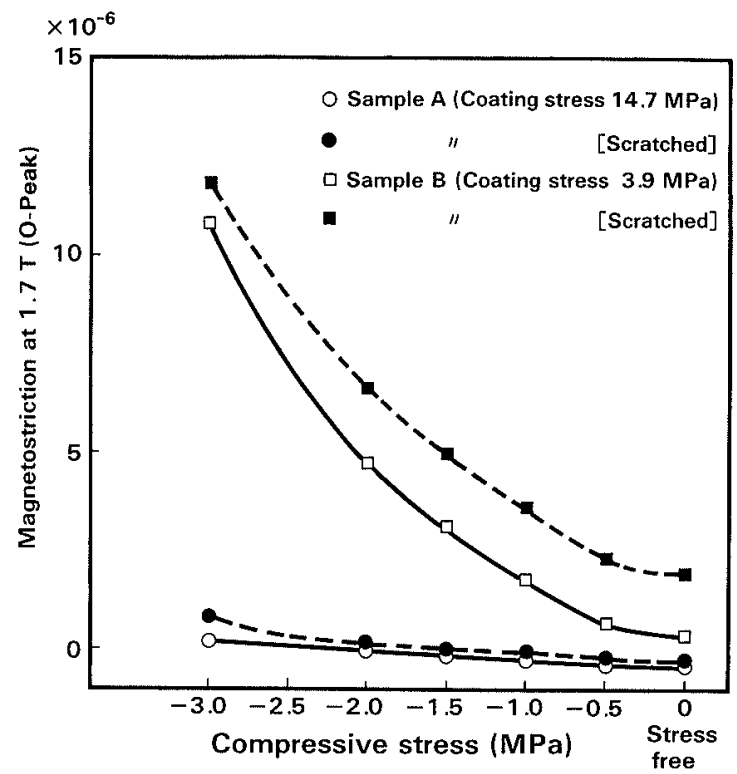

Fig. 1. Effect of compressive stress to specimen on magnetostriction $(1.7 \mathrm{~T}, 50 \mathrm{~Hz}, 0$-Peak) in different coating stress sample $\mathrm{A}$ and $\mathrm{B}$.

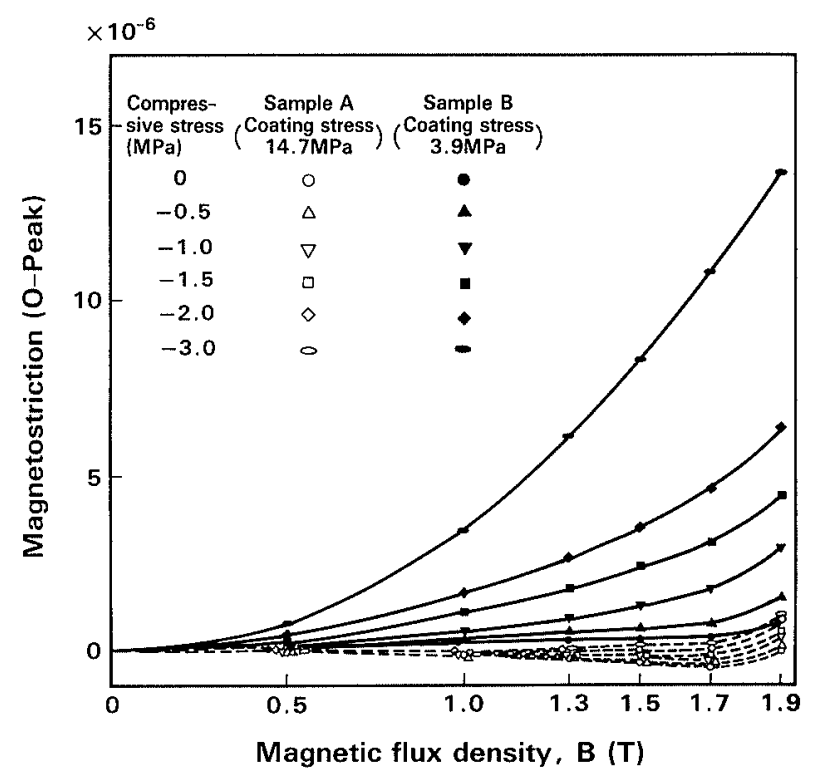

Fig. 2. Relation between magnetostriction $(50 \mathrm{~Hz})$ and magnetic flux density under stress condition in different coating sample $\mathrm{A}$ and $\mathrm{B}$.

main domains) were observed (Fig. 4(a)) except around the scratch, while moderate amount of the supplementary domains $\left(90^{\circ}\right.$ supplementary domains) which bèar $90^{\circ}$ domain wall component and, in this case, is normal to rolling direction, under $4 \mathrm{MPa}$ compressive stress (Fig. 4(b)) and much more the supplementary domains under $6 \mathrm{MPa}$ compressive stress (Fig. 4(c)). On the other hand, in sample $B$, the critical compressive stress for the occurrence of the supplementary domains was just near $1 \mathrm{MPa}$ (Fig. 5(b)) and mostly the finer supplementary domains were found even under $2 \mathrm{MPa}$ compressive stress (Fig. 5(c)). The difference in observation between sample $A$ and $B$ coincides substantially with that in the magnetostriction in Fig. 1. Scratched line on surface perpendicular to rolling direction in the test specimens contributes also as a marker to recognize the direction 


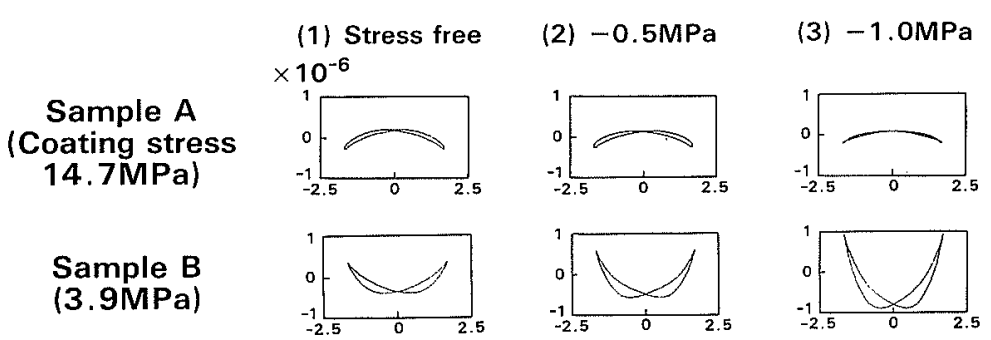

Flux density, B (T)
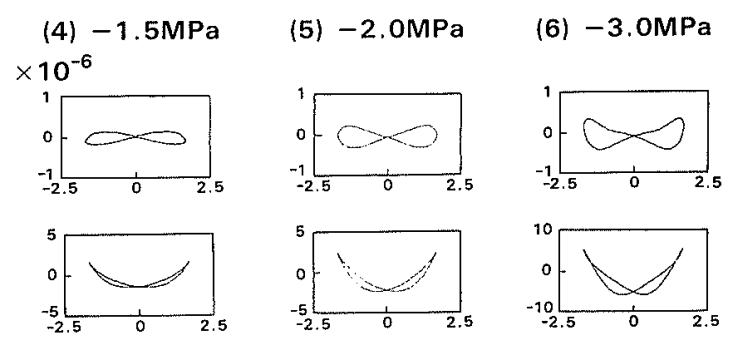

Fig. 3.

Butterfly loops of magnetostriction at $1.7 \mathrm{~T}(50 \mathrm{~Hz})$ under stress free and compressive stress conditions in different

Flux density, $B(T)$ coating stress samples.

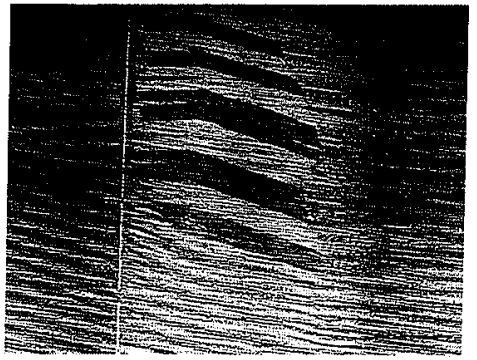

(a) Stress free

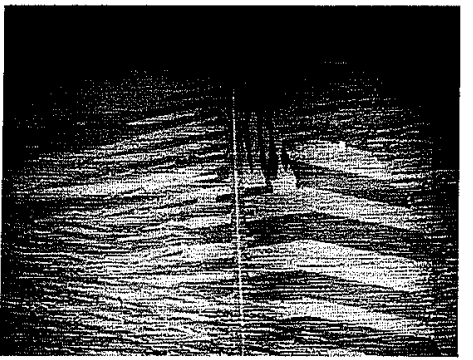

(b) Compressive stress
$4 \mathrm{MPa}$

$1 \mathrm{~mm}$

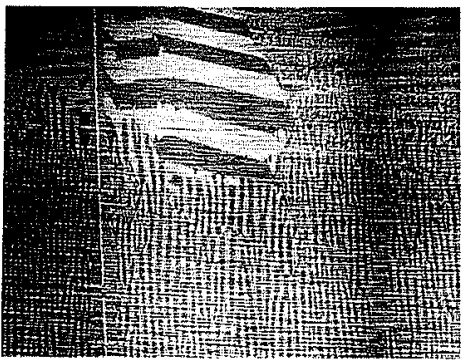

\section{(c) Compressive stress $6 \mathrm{MPa}$}

Fig. 4. Appearance of the supplementary domains under applied compressive stress to sample A (Coating stress 14.7 MPa).

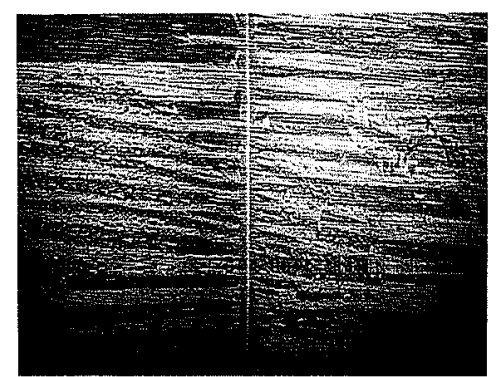

(a) Stress free

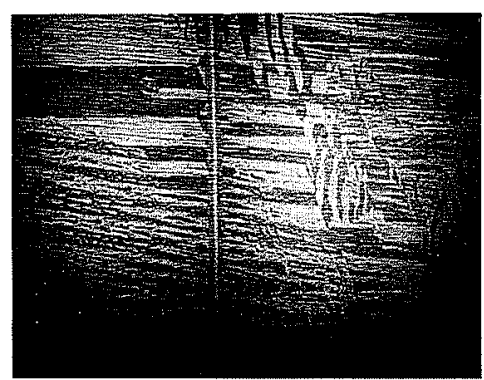

(b) Compressive stress $1 \mathrm{MPa}$

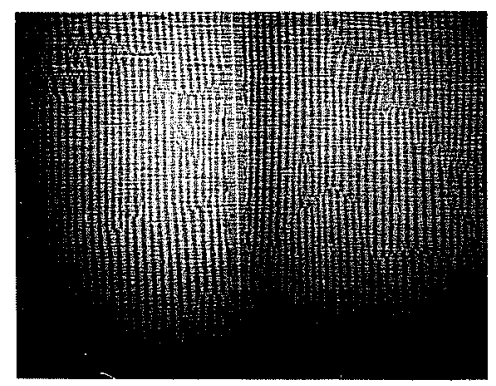

(c) Compressive stress $2 \mathrm{MPa}$

\section{$1 \mathrm{~mm}$}

Fig. 5. Appearance of the supplementary domains under applied compressive stress to sample B (Coating stress 3.9 MPa).

and scale of the magnetic domains in scanning electron microscopic observation. Along the scratched line, the triangular closure domains were observed even in the stress-free state. In sample $\mathrm{B}$, the triangular closure domains extended about $420 \mu \mathrm{m}$ from the scratched line, while about $190 \mu \mathrm{m}$ in sample A as recognized in Fig. 6. In $850^{\circ} \mathrm{C} \times 5 \mathrm{~h}$ stress relief annealed specimen, the tri- angular closure domain along the scratched line almost disappeared in either sample A or B as observed in Fig. 7. Furthermore, an incidental interesting discovery in the experiment was an observation of amalgamation of the supplementary domains occurring by applied compressive stress and the triangular closure domains along the scratched line with increasing compressive stress as 


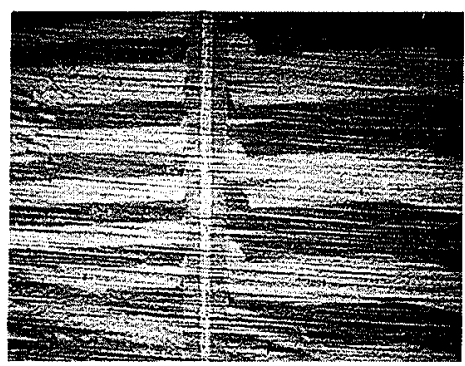

(a) Sample A (Coating stress $14.7 \mathrm{MPa}$ )

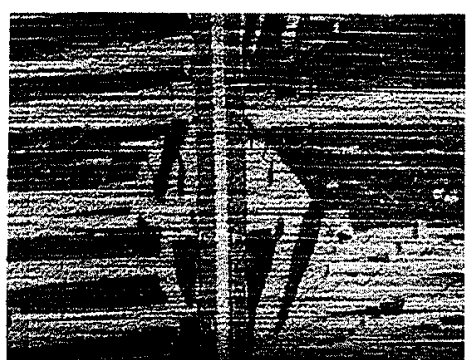

(b) Sample B (Coating stress 3.9MPa)

\section{$1 \mathrm{~mm}$}

Fig. 6. Influence on extent of the triangular closure domains from scratched line by different coating stress under applied stress free condition.

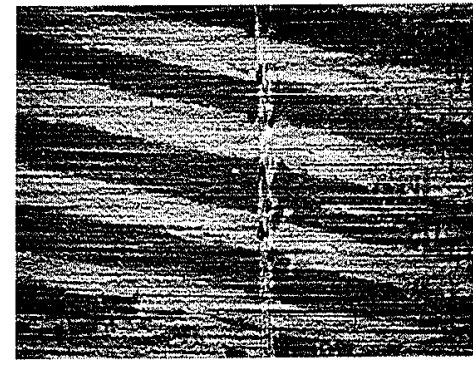

(a) Sample A

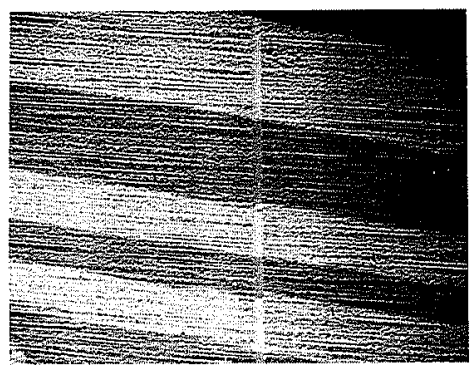

(b) Sample B

\section{$1 \mathrm{~mm}$}

Fig. 7. Disappearance of the triangular closure domains in sample A (Coating stress $14.7 \mathrm{MPa}$ ) and sample $\mathrm{B}$ (3.9 $\mathrm{MPa}$ ) after $850^{\circ} \mathrm{C} \times 5 \mathrm{~h}$ stress relief annealing.

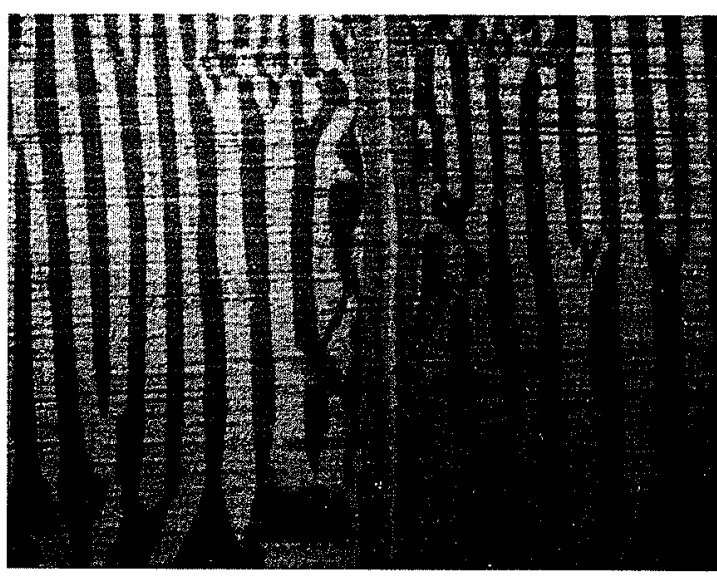

$1 \mathrm{~mm}$

Fig. 8. An amalgamation of the supplementary domains and the triangular closure domains near scratched line in sample B under applied compressive stress $4 \mathrm{MPa}$.

observed in Fig. 8.

\section{A Proposal of Theory for Stress Influence on the Magnetostriction}

It is already known that magnetostriction of steel is caused by movement of $90^{\circ}$ domain walls according to the equation $\Delta L / L=(3 / 2) \lambda\left(1-\cos ^{2} \phi\right)$ and is illustrated in Fig. 9 by Chikazumi, ${ }^{10,29)}$ where $\phi$ is the angle between

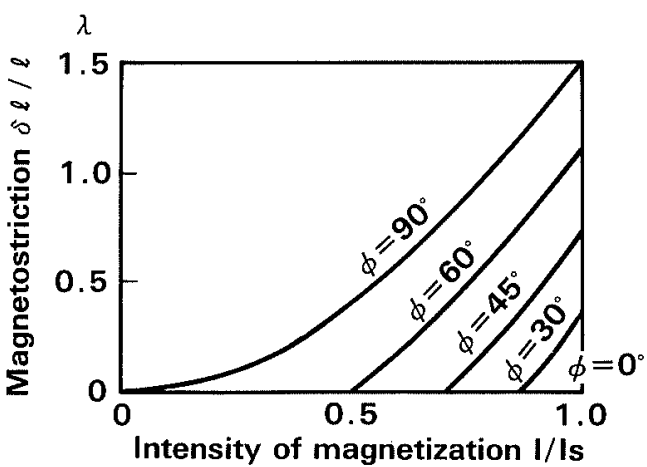

Fig. 9. Relation between magnetostriction and angle between easy magnetization axis and magnetic field (after Chikazumi ${ }^{10,29)}$ ).

the axis of easy magnetization and the direction of magnetic fields. Therefore, in the case of grain oriented silicon steel, the important factor for predicting magnetostriction is how to evaluate the conditions for the formation of the $90^{\circ}$ domain walls. Magnetoelastic energy theory has been frequently applied even for explaining the magnetostriction in grain oriented silicon steel. ${ }^{10-20)}$ Magnetostriction in grain oriented silicon steel is, however, far susceptible to influence of stress condition and, therefore, might be rather related directly to stress dynamics or strain elastic energy. As was made clear by Honda and Kaya, ${ }^{3 ; 21)}$ iron has positive magnetostrictive elongation in $\langle 100\rangle$ easy magnetization 
axis, and in the nature of thing, as experimented by Williams, ${ }^{22,23)}$ magnetization inside the domains turns toward tensile direction and forms clear domains parallel to the direction if tensile stress is applied to $\langle 100\rangle$ of the iron crystal. These studies suggest that a formation of [100] magnetic domain with spin has physically close interaction with tensile elastic elongation in $\langle 100\rangle$ direction in the strongly magnetic substance, which becomes more conspicuous in single iron crystal or grain oriented silicon steel. The fact that generally the elongation in iron crystal is dominated by strain elastic energy, needs a precise calculation about the influence of strain elastic energy in various stress conditions by using anisotropic elastic coefficients on the formation of magnetic domains. Even the positive elongation by the magnetostriction in grain oriented silicon steel under magnetic field is generated by movement of $90^{\circ}$ domain walls in the supplementary domains or the triangular closure domains existed under non-magnetic field due to stresses, as is easily recognized in Fig. 2. As indicated in the authors' observation on magnetic domain under stress field without compulsory external magnetic field in scanning electron microscope, the domains drastically changed their direction by stress condition. This suggests that strain elastic energy which dominates elastic deformation may change a direction of domains, resulting in a large influence on the magnetostriction. This yields a hypothesis that the largest strain elastic

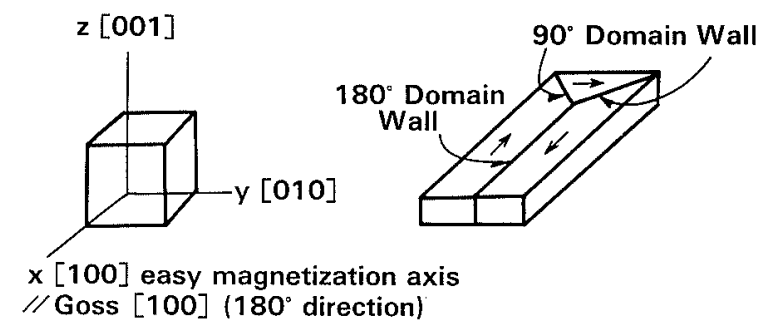

Fig. 10. Schematic drawings of iron cubic crystal and domain walls. energy of those for the three possible directions of magnetization $(x[100], y[010]$ and $z[001])$ should determine the direction of the domains under the particular stress condition. The Goss orientation texture usually has main $\left(180^{\circ}\right)$ domains parallel to $x[100]$ axis of cubic crystal. Figure $\mathbf{1 0}$ shows schematic drawings of iron cubic crystal and domain walls. Then, more physical condition for the formation of the $90^{\circ}$ supplementary domains is expressed by

$$
E_{x[100]}^{\mathrm{el}} \leqq E_{y[010](z[001])}^{\mathrm{el}}
$$

(balance of strain elastic energy

in easy magnetization axes)

As a geometrical symmetry of Goss orientation to the steel sheet, usually $E_{y[010]}^{\mathrm{el}}$ may be equal to $E_{z[001]}^{\mathrm{el}}$ in proper grain oriented silicon steel. Chikazumi ${ }^{10,29)}$ pointed out already that magnetoelastic energy associated with each axis of easy magnetization comes to differ from each other according to the application of stress, and this tends to cause the formation of a $90^{\circ}$ domain wall. By using $\sigma_{X-Y-Z}$ : stress tensor in specimen axes, $\sigma_{x-y-z}$ : stress tensor in easy magnetization axes, $\xi_{x-y-z}:$ strain tensor in easy magnetization axes, $S$ : anisotropic elastic coefficient tensor, and $T$ : tensor for coordinates transformation, the authors, by a mathematical procedure for instance by Masui, 24,35) derived strain elastic energy tensor $E_{x-y-z}^{\mathrm{el}}$. By this, strain elastic energy in easy magnetizaion axes, $E_{x[100]}^{\mathrm{el}}, E_{y[010]}^{\mathrm{el}}$ and $E_{z[001]}^{\mathrm{el}}$ was obtained as given in Table 2 including various practical stress conditions. Additionally, it may be referred to that a kind of magnetoelastic energy in each axis of easy magnetization can be also obtained by using $\sigma_{x-y-z}$ in the each axis multiplied by $\lambda_{100}$ (elongation by spontaneous magnetization) in $-(3 / 2) \lambda_{100} \sigma$, and the total elastic energy combined with both elastic energies consisting of the strain elastic energy and the abovementioned energy, is calculated easily. In this case, the total elastic energy was put instead of the strain elastic energy in the Eq. (1) for reference.

Table 2. Calculated formulas of strain elastic energy in the easy magnetization axes under various stress conditions of grain oriented silicon steel.

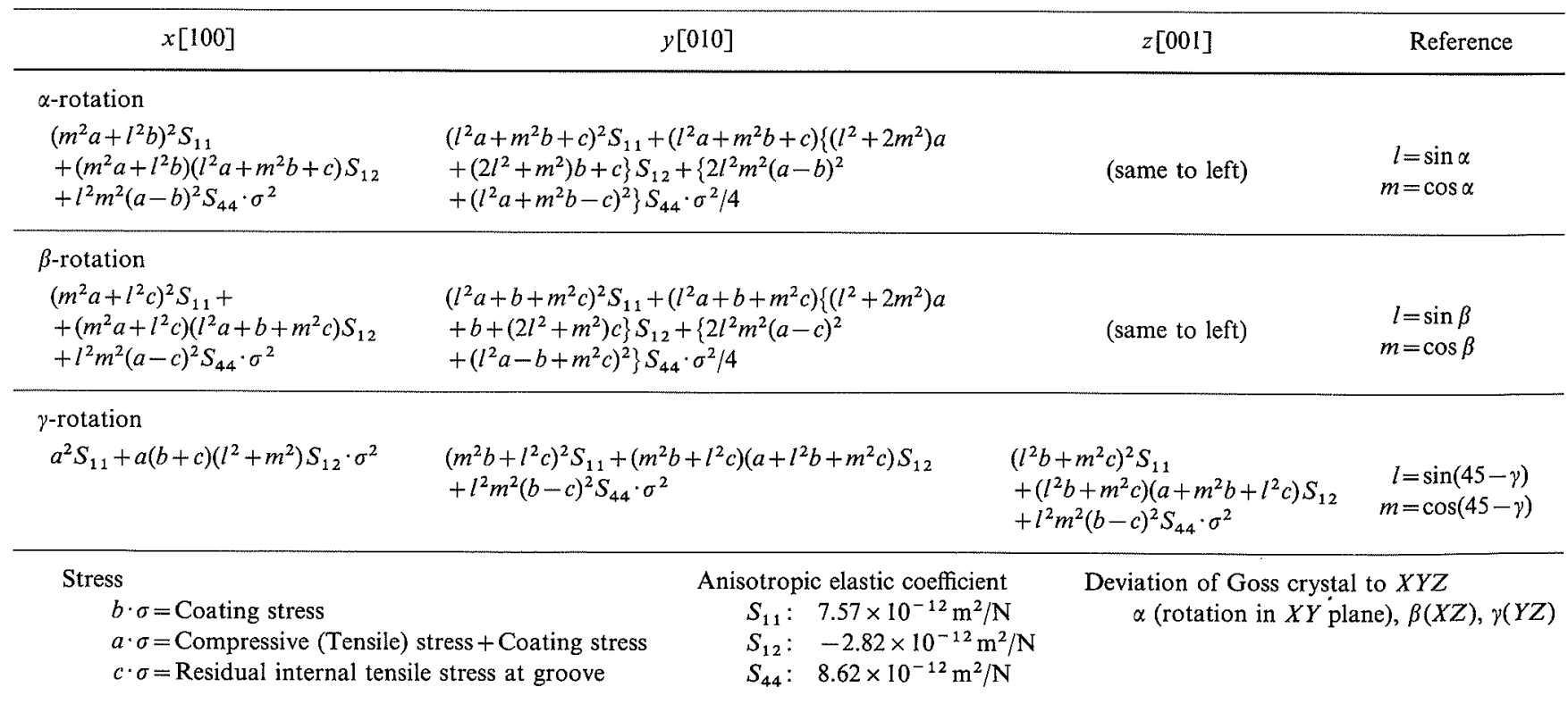




\section{Examples of Application of the Theory}

\subsection{Critical Applied Compressive Stress for the For- mation of the Supplementary Domains}

Figure 11 indicates a critical compressive stress for the formation of the supplementary domains based on the condition (1), being related with an occurrence of the magnetostriction, in different coating stress sample A and $\mathrm{B}$. This figure shows that the critical compressive stress based on the condition (1) is $3.5 \mathrm{MPa}$ (sample A) and $0.8 \mathrm{MPa}$ (sample $\mathrm{B}$ ), while in observation it is near or slightly less than $4 \mathrm{MPa}(\mathrm{A})$ and $1 \mathrm{MPa}(\mathrm{B})$ respectively as is shown in Figs. 4 and 5. This coincidence enables substantially forecasting magnetostrictive elongation as shown in Fig. 1.

Under preferable normal condition of $E_{x[100]}^{\mathrm{el}}>$ $E_{y[010](z[001])}^{\mathrm{el}}$ in grain oriented silicon steel, magnetostrictive elongation by magnetization in the rolling direction, if the specimen being set parallel to the rolling direction as usual, shall no longer take place basically, since main domains have already full elongation. On the other hand, under undesirable condition of $E_{x[100]}^{\mathrm{el}} \leqq E_{y[010](z[001])}^{\mathrm{el}}$, the supplementary domains or the triangular closure domains most deviated from the rolling direction shall inevitably rotate to the magnetic field applied in the rolling direction, being accompanied with a movement

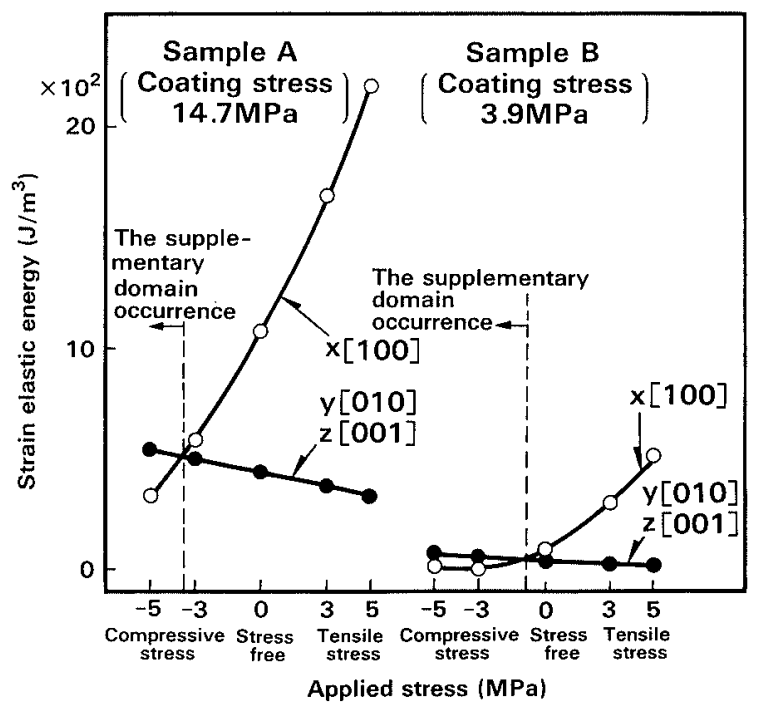

Fig. 11. Effect of coating stress on strain elastic energy in easy magnetization axes $x[100], y[010]$ and $z[001]$ under various applied stress conditions. of $90^{\circ}$ domain walls, definitely bringing about elongation proportional to the magnetic flux density, as represented in Fig. 2 (sample B).

\subsection{Extent of Triangular Closure Domains from a Scratched Line}

Fukawa ${ }^{25)}$ calculated the distribution of residual internal tensile stress normal to the surface near scratched line by FEM in grain oriented silicon steel in the case of about $40 \mu \mathrm{m}$ scratched contact width with a ball-point pen, which is nearly the same condition as the authors' experiment. By the equation in Table 2, the strain elastic energy was calculated with supposed residual internal tensile stress in different coating stress sample A and B under various applied stress conditions (Fig. 12). Therefore, by reading the critical residual internal tensile stress for the condition (1) without applied stress in sample $A$ and $B$ in Fig. 12 as $15 \mathrm{MPa}(\mathrm{A})$ and $4 \mathrm{MPa}$ (B), and inserting them into the distribution curve of residual internal tensile stress near the scratched line by Fukawa, the authors derived the extent of the triangular

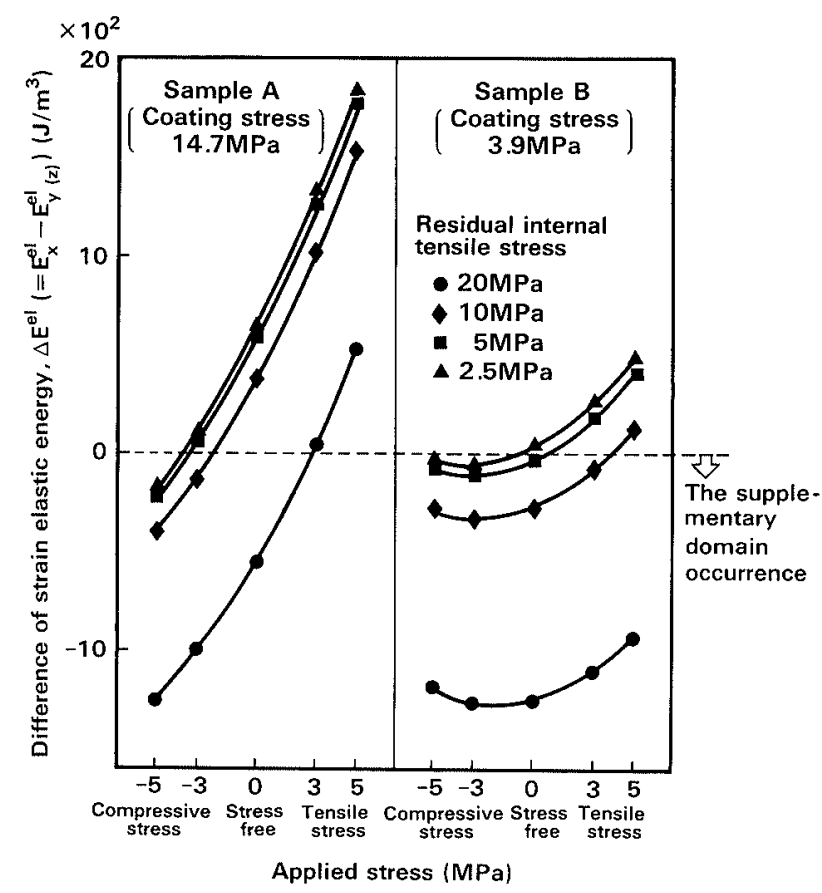

Fig. 12. Relation between the difference of strain elastic energy $\Delta E^{\mathrm{el}}\left(=E_{x}^{\mathrm{cl}}-E_{y(z)}^{\mathrm{cl}}\right)$ in easy magnetization axes and residual internal tensile stress near scratched line under various applied stress conditions to different coating stress sample $\mathbf{A}$ and $\mathbf{B}$.

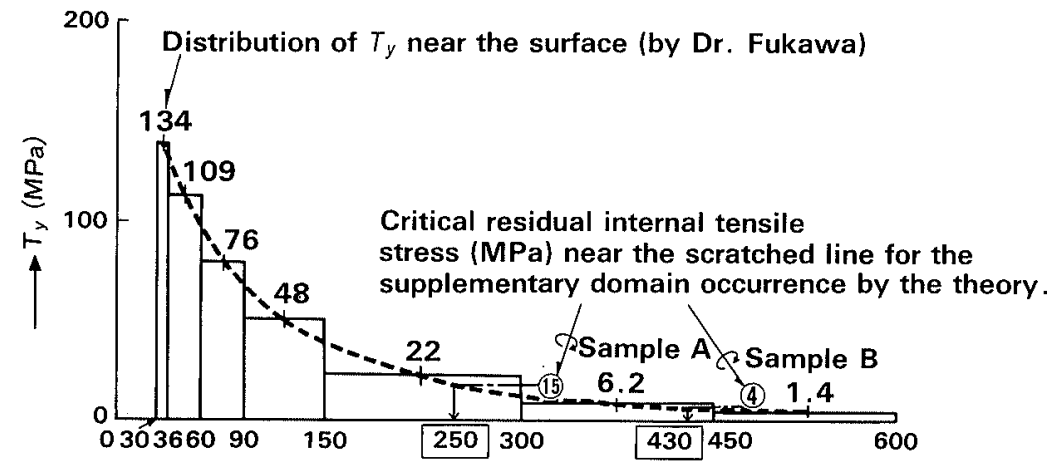

Distance from the scratched line in the rolling direction $(\mu \mathrm{m})$
Fig. 13.

Estimation of extent of the triangular closure domains occurrence from scratched line by the condition $\Delta E^{\mathrm{el}}\left(=E_{x}^{\mathrm{el}}-E_{y(z)}^{\mathrm{el}}\right) \leqq 0$ using a distribution of residual internal tensile stress $T_{y}$ calculated by Fukawa ${ }^{25}$ ) in the case of $40 \mu \mathrm{m}$ contact width on surface with a ball-point pen. 


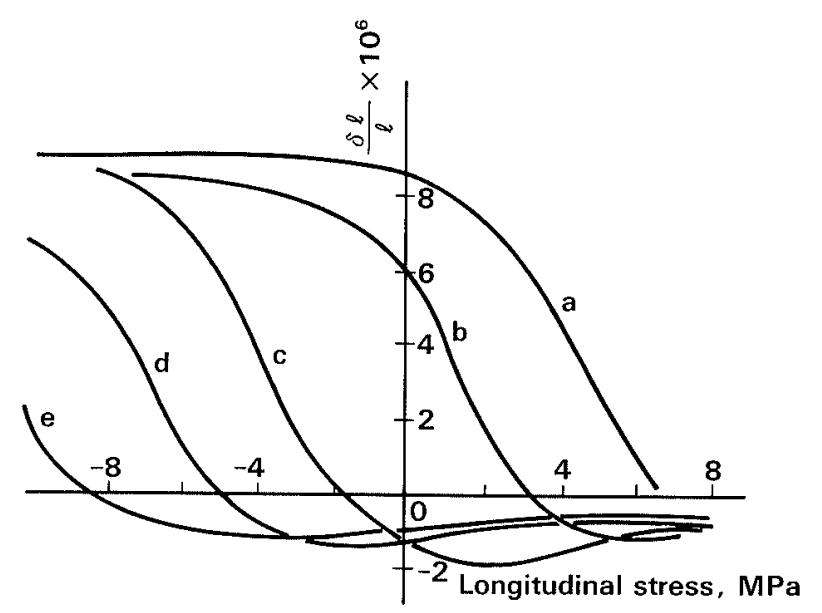

Variation of fundamental component of magnetostriction $(1.5 \mathrm{~T}, 50 \mathrm{~Hz})$ in grain oriented silicon iron under longitudinal stress with transverse stress of (a) + 10MPa, (b) +6MPa, (c) zero, (d) -6MPa, (e) $-10 \mathrm{MPa}$

Fig. 14. Effect of superimposed longitudinal and transverse stress on magnetostriction of grain oriented silicon steel by Moses. ${ }^{12,13)}$

closure domains from the scratched line without applied stress in sample A and B as 250 and $430 \mu \mathrm{m}$ respectively as denoted in Fig 13. These values are substantially comparable with the experimental values of 190 (A) and 420 (B) $\mu \mathrm{m}$ seen in Fig. 6. Furthermore, an incidental interesting discovery in the experiment was the amalgamation of the supplementary domains occurring with compressive stress applied and the triangular closure domains along the scratched line, which was observed with increasing compressive stress in scanning electron microscope as seen in Fig. 8. This observation might support the concept of the generalization for the formation of $90^{\circ}$ domain walls by the condition (1) under various stress states, resulting in unification of the magnetostriction by the two causes. Disappearance of the triangular closure domains without applied stress in the specimens fully stress-relief annealed (Fig. 7) can be reasonably recognized due to a relation of $E_{x[100]}^{\mathrm{el}}>$ $E_{y[010](z[001])}^{\mathrm{el}}$ by supposing the residual internal tensile stress near the scratched line to be zero in Fig. 12.

\subsection{Prediction of the Magnetostriction in Bi-axial Stress Condition by Moses}

Moses et $a .^{12,13)}$ studied the magnetostriction in the rolling direction under bi-axial longitudinal and transverse stress condition in grain oriented silicon steel and got important experimental data represented in Fig. 14. Similar calculation to the equations in Table 2 , was carried out and the result is shown in Fig. 15. The region $\Delta E^{\mathrm{el}}=E_{x[100]}^{\mathrm{el}}-E_{y[010](z[001])}^{\mathrm{el}} \leqq 0$ indicates the formation of the supplementary domains, which is predicted by the theory based on the condition (1), and shows an area of the magnetostriction. The calculation in Fig. 15 fairly explains the experimental result in Fig. 14 by Moses et al. The authors also studied substitution of the total elastic energy, i.e. sum of the strain elastic energy and $-(3 / 2) \lambda_{100} \sigma$ in easy magnetization axes, into the strain elastic energy in the condition (1) and got substantially

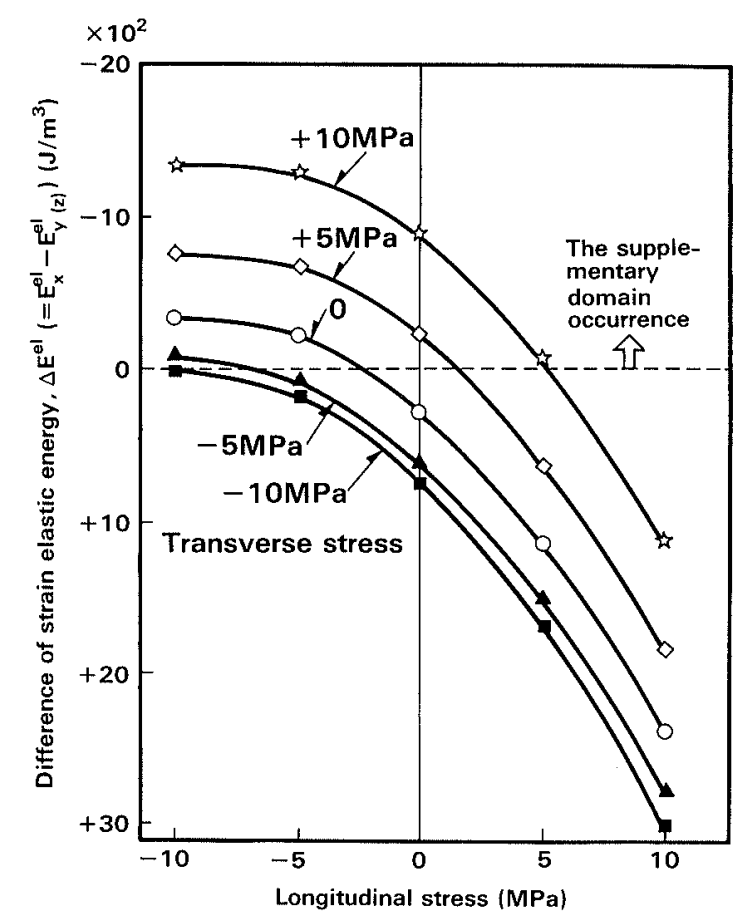

Fig. 15. Effect of superimposed longitudinal and transverse stress on the difference of strain elastic energy between easy magnetization axis $x[100]$ and $y[010](z[001])$ of grain oriented silicon steel with coating stress of $10 \mathrm{MPa}$.

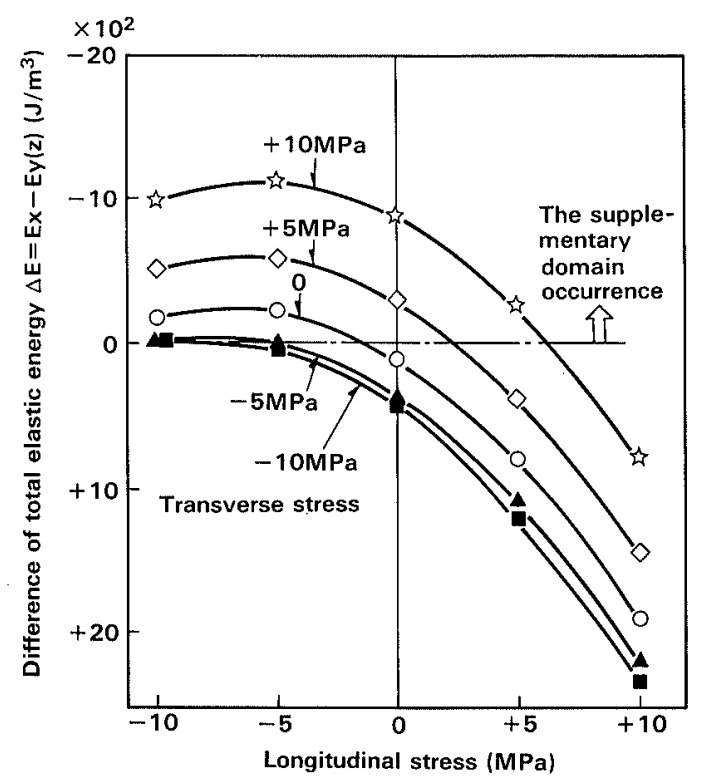

Fig. 16. Effect of superimposed longitudinal and transverse stress on the difference of total elastic energy between easy magnetization axis $x[100]$ and $y[010](z[001])$ of grain oriented silicon steel with coating stress of $10 \mathrm{MPa}$.

a similar fruitful result as shown in Fig. 16. This proves a determinative effect and practical importance of the strain elastic energy on the formation of the supplementary domains or the triangular closure domains in grain oriented silicon steel, as supported by observation in scanning electron microscope under the stress conditions.

Other than the above examples, the formulae derived from the condition (1) suggest a solution to a mazed 
Table 3. Concepts of low magnetostriction grain oriented $3 \%$ silicon steel.

\begin{tabular}{|c|c|c|}
\hline Properties & Effects & Technologies \\
\hline $\begin{array}{l}\text { High tension stress } \\
\text { coating }\end{array}$ & $\begin{array}{l}\text { Steel bearable to compressive stress in rolling direction. The } \\
\text { higher coating tension stress, the less compatible with the } \\
\text { condition ( } 1)\left(E_{x[100]}^{\mathrm{el}} \leqq E_{y[010](z[001])}^{\mathrm{e}]}\right) \text {, suppressing a forma- } \\
\text { tion of the supplementary domains. }\end{array}$ & $\begin{array}{l}\text { - High tension glass film } \\
\text { - High tension insulation coating film } \\
\text { - Other wet process coating film } \\
\text { - Dry process coating film (PVD, CVD etc.) }\end{array}$ \\
\hline $\begin{array}{l}\text { High permeability } \\
\text { material }\end{array}$ & $\begin{array}{l}\text { Steel with less deviation from just Goss texture }(\{110\}\langle 001\rangle) \text {. } \\
\text { The less deviation, the less inferior magnetostriction due to } \\
\text { rotation of less the supplementary domains or deviated domains } \\
\text { in large magnetic field. }\end{array}$ & $\begin{array}{l}\text { Optimum manufacturing process for grain } \\
\text { oriented silicon steel (Hot rolling, Cold } \\
\text { rolling reduction, Inhibitor control in } \\
\text { secondary annealing, etc.) }\end{array}$ \\
\hline
\end{tabular}

causation among production factors in grain oriented silicon steel and causes through transformer fabrication and operation for noise problem of transformer.

Based on these concepts, low magnetostriction grain oriented $3 \%$ silicon steel shall be practically developed. Some of the principal ideas are proposed in Table 3. Sample A in the experiment, for instance, granted having been subjected to some experimental treatment to a certain degree, could be said typical of the low noise steels.

\section{A Comparison of the Theory with the Previous Magnetostriction Studies}

It is known that a formation of magnetic domain is dominated by such energies as magnetostatic energy, domain wall energy, magnetic anisotropy energy and magnetoelastic energy. ${ }^{10,11,26-32)}$ It has been also explained ${ }^{10,26-29)}$ that the magnetoelastic energy is nothing else but the magnetic anisotropy energy under stress loading and the most dominating energy of them to the magnetostriction. The magnetostatic energy $^{10,11,29,30)}$ is the energy of magnetic field generated by magnetic pole due to spontaneous magnetization in strongly magnetic crystal and has about an order of $10^{3} \mathrm{~J} / \mathrm{m}^{3}$. The domain wall energy ${ }^{10,11,29,30)}$ is energy of walls between magnetic domains and has a very small value of about an order of $10^{\circ} \mathrm{J} / \mathrm{m}^{3}$. On the other hand, the magnetic anisotropy energy ${ }^{10,11,29,30)}$ is the energy which directs the spontaneous magnetization in strongly magnetic crystal, generated by a change of internal energy according to change of direction of the spontaneous magnetization and has about an order of $10^{3} \mathrm{~J} / \mathrm{m}^{3}$. On the point of an order of energy magnitude, the magnetic anisotropy energy or the magnetoelastic energy is important as well as the magnetostatic energy in a research of the magnetic domains. As far as the magnetostriction is dominated, however, in the previous studies, ${ }^{10-12,26-29)}$ it is rather popular that the magnetostriction is exclusively related to the magnetoelastic energy with a balance of other energies. It is recognized ${ }^{10,26-29)}$ that the magnetic anisotropy energy is dealt with as just the magnetoelastic energy under stress loading, being explained by means of the Neel's spin pair model. ${ }^{32)}$ First of all, therefore, the author's theory has to be discussed in comparison with the magnetoelastic energy under the stress. Kaya ${ }^{28)}$ explained the formation of the $\langle 100\rangle$ domains by means of the stress potential energy which was defined as an energy tensor of stress and strain. This definition resembles to the authors' one even though the former did neither explain the formation of the supplementary domains under the complicated stress conditions and was nor followed by practical experiments. Becker and Döring, ${ }^{26)}$ and Chikazumi ${ }^{10,29)}$ introduced the magnetoelasic energy under stress loading using the Néel's model. The expression of the magnetoelastic energy is, however, quite same with the stress potential energy by Kaya. Chikazumi ${ }^{10,29)}$ also suggested a possibility of the formation of the supplementary domains under a simple stress loading in iron crystal for the reason that the magnetoelastic energies in three axes of easy magnetization come to differ from each other by the stress. This suggestion is important in the study of the magnetostriction even if the work was rather qualitatively examined. Additively, it is also interesting in the work that the magnetoelastic energy under stress loading seems to be expressed as a product between the stress and the magnetostrictive elongation under no stress loading. This suggests that the strain elastic energy by stress loading in the strongly magnetic substance such as a single iron crystal or the Goss oriented grain oriented silicon steel, is not only hardly discussed separatedly from the magnetic energies or magnetic properties, but also is even intrinsically same with the magnetoelastic energy as experimentally demonstrated by Williams. ${ }^{22,23)}$ The authors'theory by the condition (1) is based on this concept and, however, still seems to need, in future work, a consideration to take in the magnetostatic energy to make the prediction with higher accuracy. As shown in Figs. 4 and 5, domain width of the supplementary domains decreased with increase of compressive stress, which might be possibly explained by a change of the magnetostatic energy. Regardless of this fact, it seems that the magnetostriction, however, was mostly explained by the condition (1) based on the strain elastic energy in the experiments, which might be probably understood by Fig. 9 in which the magnitude of the magnetostriction is mainly dominated rather by the angle between the direction of axis of easy magnetization and that of magnetic field than the width or the number of the domains. Furthermore, the authors shall have an interest in the interaction between the dislocation and the stress field under magnetic field with the manner cultivated by Krönmuller, Seeger and Umakoshi ${ }^{33,34)}$ even in the magnetostriction if the steel is heavily stressed. 


\section{Conclusion}

Causes of noise emitted from transformer core are more or less attributed to magnetostriction of the core materials. The magnetostriction in grain oriented silicon steel is fatally influenced by stress condition on the products. A hypothesis on a critical condition for the formation of the $90^{\circ}$ supplementary domains or the triangular closure domains at groove which determinatively cause the magnetostriction in grain oriented silicon steel, as expressed by $E_{x[100]}^{\mathrm{el}} \leqq E_{y[010](2[001])}^{\mathrm{el}}$ (balance of 'strain elastic energy in easy magnetization axes) was ascertained by elaborate experiments. By the condition, occurrence of the magnetostriction under superimposed stress condition such as influence of applied stress to specimen and residual internal tensile stress near scratched line with a ball-point pen, effect of coating stress as well as the magnetostriction under bi-axial longitudinal and transverse stress to specimen by Moses, could be substantially explained in the mathematically derived calculation. At the end, additionally, it was referred to how a concept on low magnetostriction silicon steel should be based on the magnetostriction concept.

\section{REFERENCES}

1) N. P. Goss: U.S.P. 1,965,559, (1934).

2) S. Taguchi and A. Sakakura: U.S.P. 3,159,511 (1964).

3) K. Honda and S. Kaya: Sci. Rep. Tohoku Univ., 15, (1926), 721

4) W. F. Barrett, W. Brown and R. A. Hadfield: Sci. Trans. Roy. Dublin Soc., 7 (1900), 67.

5) E. Gumlich and P. Goerens: Trans. Faraday Soc., 8 (1912), 98.

6) R. A. Hadfield: U.S.P. 745,829 (1903).

7) M. F. Littmann: U.S.P. 3,764,406 (1973).

8) T. D. Yensen: Phys. Rev., 6 (1915), 404.

9) V. W. Carpenter and J. M. Jackson: U.S.P. 2,287,467 (1942).

10) S. Chikazumi: Physics of Magnetism, John Wiley \& Sons, Inc., New York, (1964), 152.

11) S. Taguchi: The Electric Magnetic Steel (Japanese), Nippon Steel, (1979), 86.

12) A. J. Moses: IEEE Trans. Magn., MAG, 15 (1979), 1575.

13) A. J. Moses and P. S. Phillips: IEEE Trans. Magn., MAG, 14 (1978), 353.

14) A. J. Moses, S. M. Pegler and J. E. Thompson: Proc. IEE, 119 (1972), 1222

15) S. M. Pegler: AIP Conf. Proc., 24 (1974), 718.

16) W. D. Corner and J. J. Mason: Brit. J. Appl. Phys., 15 (1964), 709.

17) A. J. Moses and E. C. Pike: Proc. IEE, 124 (1977), 583.

18) W. M. Swift and G. F. Wolfe: IEEE Trans. Magn., MAG, 10 (1974), 195.

19) R. Perryman: J. Phys. D, 8 (1975), 1901.

20) T. Yamamoto, S. Taguchi, A. Sakakura and T. Nozawa: IEEE Trans. Magn., MAG, 8 (1972), 677.

21) S. Kaya and H. Takaki: Sci. Rep. Tohoku Univ., (1936), 314.

22) H. J. Williams, R. M. Bozorth and W. Shockley: Phys. Rev., 75 (1949), 115.

23) H. J. Williams and W. Shockley: Phys. Rev., 75 (1949), 178.

24) H. Masui: Kimitsu Research Report (Japanese), Nippon Steel, (1975), 56

25) K. Fukawa: J. Jpn. Inst. Met, 45, No 4 (1981), 384.

26) R. Becker and W. Döring: Ferromagnetismus, Springer, Berlin, (1939), 482.

27) R. M. Bozorth: Ferromagnetism, IEEE Press, (1993), 595.

28) S. Kaya: Strong Magnetism (Japanese), Iwanami, Japan, (1951), 103.

29) S. Chikazumi: Physics of Ferromagnetism, Vol. II (Japanese), Syokabo, Japan, (1984), 106.

30) J. W. Shilling and G. L. Houze: IEEE Trans. Magn., MAG, 10
(1974), 195.

31) W. Heisenberg: Z. Phys., 49 (1928), 619

32) L. Néel: J. Phys. Radium, 15 (1954), 225.

33) H. Krönmuller and A. Seeger: J. Phys. Chem. Solids, 18 (1961), 93.

34) Y. Umakoshi and H. Krönmuller: Phys. Status Solidi (a), 68 (1981), 159.

35) H. Masui: in preparation, (1994).

\section{Appendix}

Evaluation of the magnetostriction through computeraided calculation method which deals with an equation of stress, shall be based on the condition (1). Application of the condition (1) shall be generalized by mathematical procedure. Usually, Goss orientation texture $\{110\}\langle 001\rangle$ has more or less a slight deviation along or around the rolling direction generally parallel to magnetostriction test specimen direction. Figure 17 denotes a definition of deviation of Goss orientation crystal from the specimen. Stress imposed on the silicon steel is schematically drawn in Fig. 18 as an example. Computing method is rather simple, granted that there is slight difference in detail from the given conditions such as a type of stress imposed on the steel and the deviation of Goss orientation crystal, etc. It should be better for understanding to describe an introduction of the equation as an example. Computing method is attributed to derivation of strain elastic energy in easy magnetization axes, $E_{x[100]}^{\mathrm{el}}, E_{y[010]}^{\mathrm{el}}$ and $E_{z[001]}^{\mathrm{el}}$. If definition of stress is put in such as $a \cdot \sigma=$ compressive (tensile) stress + coating stress, $b \cdot \sigma=$ coating stress and $c \cdot \sigma=$ residual internal tensile stress at groove (scratch) and $\sin \alpha=L, \cos \alpha=$ $m(\alpha$ : rotational deviation of Goss orientation crystal $\{110\}\langle 001\rangle$ in $X Y$ plane of the specimen, i.e. plane of steel sheet (Fig. 17)), strain elastic energy was obtained mathematically by means of stress tensor and strain

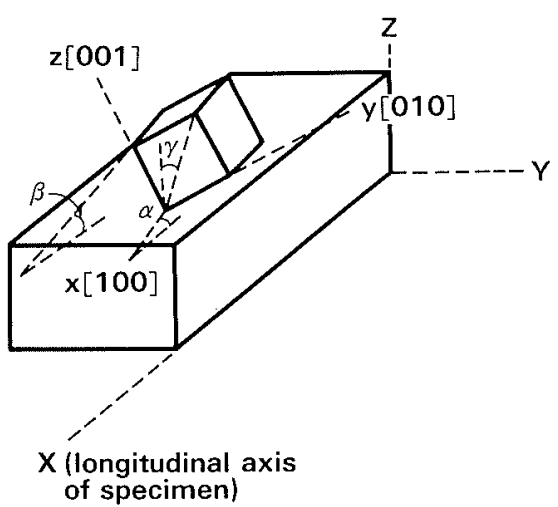

Fig. 17. Relation between coordinates $(x, y, z)$ of cubic crystal and those $(X, Y, Z)$ of specimen.

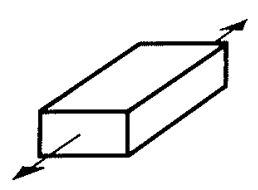

Applied stress

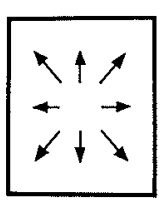

Coating stress

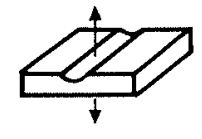

Residual internal tensile stress at groove
Fig. 18. Schematic drawings of various stress conditions occuring in grain oriented silicon steel. 
tensor as follows.

Stress tensor was obtained as follows.

$$
\begin{aligned}
\sigma_{x-y-z}= & T^{-1} \sigma_{X-Y-z} T=(\sigma / 2) \cdot\left[\begin{array}{ccc}
\sqrt{2} m & \sqrt{2} L & 0 \\
-L & m & 1 \\
L & -m & 1
\end{array}\right] \\
& \cdot\left[\begin{array}{lll}
a & 0 & 0 \\
0 & b & 0 \\
0 & 0 & c
\end{array}\right] \cdot\left[\begin{array}{ccc}
\sqrt{2} m & -L & L \\
\sqrt{2} L & m & -m \\
0 & 1 & 1
\end{array}\right] \\
= & (\sigma / 2) \cdot\left[\begin{array}{cc}
-\sqrt{2} L m a+\sqrt{2} L m b \\
2 m^{2} a+2 L^{2} b \\
\sqrt{2} L m a-\sqrt{2} L m b \\
& -\sqrt{2} L m a+\sqrt{2} L m b \\
& L^{2} a+m^{2} b+c \quad-L^{2} a-m^{2} b+c \\
& -L^{2} a-m^{2} b+c \quad L^{2} a+m^{2} b+c
\end{array}\right]
\end{aligned}
$$

(A-1)

( $T:$ tensor for coordinates transformation)

Strain tensor was obtained as follows.

$$
\begin{aligned}
& \xi_{x-y-z}=S \sigma_{x-y-z}=(\sigma / 2) \\
& {\left[\begin{array}{cccccc}
s_{11} & s_{12} & s_{12} & 0 & 0 & 0 \\
s_{12} & s_{11} & s_{12} & 0 & 0 & 0 \\
s_{12} & s_{12} & s_{11} & 0 & 0 & 0 \\
0 & 0 & 0 & s_{44} & 0 & 0 \\
0 & 0 & 0 & 0 & s_{44} & 0 \\
0 & 0 & 0 & 0 & 0 & s_{44}
\end{array}\right] \cdot\left[\begin{array}{c}
2 m^{2} a+2 L^{2} b \\
L^{2} a+m^{2} b+c \\
L^{2} a+m^{2} b+c \\
-L^{2} a-m^{2} b+c \\
\sqrt{2} L m(a-b) \\
-\sqrt{2} \operatorname{Lm}(a-b)
\end{array}\right]}
\end{aligned}
$$

( $S:$ anisotropic elastic coefficient tensor)

Strain elastic energy tensor was derived from the stress tensor and the strain tensor as follows.

$$
\begin{aligned}
& E_{x-y-z}^{\mathrm{el}}=\sigma_{x-y-z} \xi_{x-y-z}=(\sigma / 2)^{2} \\
& \qquad\left[\begin{array}{ccc}
4\left(m^{2} a+L^{2} b\right)^{2} s_{11}+4\left(m^{2} a+L^{2} b\right) & & \\
\left(L^{2} a+m^{2} b+c\right) s_{12}+4 L^{2} m^{2}(a-b)^{2} s_{44} & & \\
\cdots & \ldots & \ldots \\
\cdots & \ldots & \ldots
\end{array}\right]
\end{aligned}
$$

Thus, $E_{x[100]}^{\mathrm{el}}, E_{y[010]}^{\mathrm{el}}$ and $E_{z[001]}^{\mathrm{el}}$ are worked out as given in Table 2. The condition (1) is fundamental formula for predicting magnetostriction in grain oriented silicon steel, being applicable to any superimposed stress conditions on the steel, even such practical case as compressive stress normal to sheet surface plane, residual internal tensile stress at scratched groove and bi- or tri- axial superimposed stress as well. 